\title{
ON THE ROLE AND IMPACT OF ELECTROMAGNETIC FIELDS IN ECOSYSTEMS
}

\author{
L. BRIZHIK \\ Bogolyubov Institute for Theoretical Physics, Kiev, Ukraine.
}

\begin{abstract}
Role and effects of electromagnetic field in living organisms and ecosystems are discussed within a nonlinear mechanism of charge transport processes that take place during respiration and photosynthesis, as well as in hydrogen-bonded chains of water molecules. Such mechanism is based on the account of the interaction between charges and induced by the distortions of macromolecules or water chains, which results in the self-trapping of charges in soliton states. It is shown that electrosolitons have some characteristic frequency, with respect to which periodic electromagnetic fields have resonant effect on their dynamics and stability. At certain conditions unbiased (with zero mean-value) periodic electromagnetic radiation can cause drift of solitons along molecular chains, and, therefore, affect charge transport processes. Such drift of particles in unbiased fields is known as ratchet phenomenon. Similar dynamics can be caused by stochastic fields and takes place in water chains present in interfacial water in cells, in coherence domains of water in the atmosphere and oceans. These results can explain the mechanism of impact of the electromagnetic solar activity on metabolism of living organisms and functioning of ecosystems. Moreover, it provides the mechanism for the formation of ecosystems via field-mediated exchange of information between the ecosystem elements and existence of the hierarchy of ecosystems. This study also indicates a possible danger of the electromagnetic smog on ecosystems.

Keywords: charge transport, coherence, ecosystems, electromagnetic field, electromagnetic smog, radiation, ratchet phenomenon, soliton, water.
\end{abstract}

\section{INTRODUCTION}

The electromagnetic field plays an essential role in the matter formation and its properties, both in-animated and alive, as well as in complex systems comprising them both, such as ecosystems. Electromagnetic field (EMF) is the main tool of information storage and transfer not only in modern technologies, but in the living processes in general. Till late 80s of the last century, EMF was studied and technologically applied in linear systems whose optical properties almost do not depend on the field intensity. Moreover, in most cases EMF was assumed to be an external field for the problems studied. This is often the case, indeed. Nevertheless, there is a large class of systems that change the properties of the field itself, and systems with endogenous EMF, when such a field is an intrinsic property of the system and is dynamically self-consistent with it. Living organisms and ecosystems are good examples of those. Therefore, the study of the effects of deterministic and stochastic EMFs on charge and information transfer processes is of major importance.

In the present paper we study the effects of an external EMF on nonlinear charge transport processes in living organisms and water systems. Such a nonlinear mechanism is based on the account of the interaction between charges and induced by the distortions of the corresponding macromolecules or water chains. This interaction results in the self-trapping of charges in soliton states which are localized solitary waves whose dispersion is compensated by the nonlinearity, due to which such waves posses exceptional stability with respect to interaction not only with similar solitons, but also with respect to effects of external fields. This mechanism is discussed in Section 2. In Section 3 it is shown that solitons that carry charges (electrosolitons) have some characteristic frequency, which determines resonant character of the influence of a periodic electromagnetic field on their dynamics and stability. In their turn, electrosolitons themselves emit EM radiation, slowly decaying with distance. Exchange by 
self-induced EM radiation results in synchronization of charge transport processes and provides the mechanism for the long-range exchange with information that can result in the formation of hierarchy of complex systems up to ecosystems. In Section 4 we show that at certain conditions unbiased (with zero mean-value) periodic in time electromagnetic radiation can cause drift of electrosolitons, and, therefore, it affects charge and information transfer processes. Such drift of particles in unbiased fields is known as ratchet phenomenon. Similar dynamics can be caused by stochastic fields and takes place in water chains present in interfacial water in cells, in coherence domains of water in the atmosphere and oceans. In Conclusions, we show that these results can explain the mechanism of correlations in the electromagnetic solar activity and biological activity of living organisms and functioning of ecosystems. Moreover, it provides the mechanism for the formation of ecosystems via field-mediated exchange of information between the ecosystem elements and existence of the hierarchy of ecosystems. This study also indicates a possible impact of solar activity on ecosystems and possible danger of the electromagnetic smog even of low intensities.

\section{CHARGE TRANSPORT PROCESSES MEDIATED BY ELECTROSOLITONS}

In living organisms, oscillating EMF not only accompanies biochemical processes, but it provides their metabolism as a regulating and controlling tool in general. No surprise that external EMF affects such systems, that the corresponding bioeffects are very complicated and intensity- and frequency-dependent. In this paper we study the effect of EMF on charge transport in nonlinear systems, such as living organisms or coherence domains of water. In particular, charge transport accompanies redox process in both organisms with photosynthetic and respiratory systems. In the redox processes certain parts of the charge transport chain (so called Krebs cycle) take place along macromolecular enzymes that form alpha-helical conformation consisting of three almost parallel polypeptide chains, stabilized by hydrogen bonds along them (Lehninger, [1]). According to Davydov [2], charge transport along these chains is provided by soliton mechanism, when electrons released at the initial stage of the photosynthesis or respiration, cause distortion of the macromolecule and get self-trapped in soliton states. Worth to recall here that the term 'soliton' means a particular type of solitary wave solution of a nonlinear wave equation, when the dispersion in the system and nonlinearity compensate each other. As a result, solitons are exceptionally stable, they propagate without energy dissipation for emission of vibrational modes in macromolecules, without change of their velocity and shape and are stable with respect to interaction with other similar solitons (see, e.g., Dauxois and Peyrard [3]).

Another example of systems supporting nonlinear charge transport are hydrogen bonded chains of water molecules. Usually these latter are considered as ice-like structures, existing at low temperatures. This is not the only case, since it is well established that water in biological systems is in the so called interfacial phase which is highly structured and/or exists in micropores formed by biological tissues, and also is structured due to the pore walls (see Pollack [4], Voeikov [5], Huang [6], Del Giudice et al. [7]). In both cases water forms hydrogen-bonded chains along which protons can propagate in the form of solitons, as has been shown by Antonchenko, Davydov, and Zolotaryuk [8]. Moreover, unique properties of water result in the formation at certain conditions of coherence domains in it where water molecules and EMF are mutually bound, which also stabilizes oriented water structures in such coherence domains (Preparata [9], Del Giudice et al [10]). One can expect that the oscillating EMF can affect such elongated hydrogen-bonded water structures if their life-time is bigger than the period of the field.

Quasiparticles (electrons, holes, protons, molecular vibrations) in the mentioned above systems are described by systems of coupled nonlinear equations for the quasiparticle wave function and chain distortion. Under certain conditions such system of equations can be reduced to the Nonlinear 
Schrodinger equation with cubic nonlinear term, that accounts for the self-consistent potential well created by the distortion of the molecular chain by the quasiparticle itself:

$$
i \hbar \frac{\partial \Psi_{n}(t)}{\partial t}=-J\left(\Psi_{n+1}+\Psi_{n-1}-2 \Psi_{n}\right)-2 J g\left|\Psi_{n}\right|^{2} \Psi_{n}
$$

Here $\Psi_{n}(t)$ is the wave function of a quasiparticle (below we will use term 'electron' for simplicity), $J$ is the resonant (exchange) interaction of electrons on nearest sites, $g$ is the nonlinearity constant, i.e. coupling between the electron and chain distortion.

Equation (1) in the leading order has a soliton solution

$$
\psi_{n}(t)=\phi_{n}(t) \exp (i Q n-i E t / \hbar)
$$

with the spatially localized envelope

$$
\phi_{n}(t)=\sqrt{\frac{\mu}{2}} \frac{1}{\cosh \{\mu[n-\zeta(t)]\}}
$$

that characterizes the amplitude of the probability of the charge presence on the $n$-th site in the molecular chain at time moment $t$. Here $Q$ is soliton wave number, $\varsigma(t)$ is its center of mass coordinate, and $E$ is the energy. The dimensionless constant $\mu$ characterizes the area of electrosoliton localization along the polypeptide chain and is determined by electron-phonon coupling constant, $\chi$, exchange energy of interaction between the nearest neighbours, $J$, and elasticity constant of hydrogen bonds, $w$ :

$$
\mu=\frac{g}{\left(1-s^{2}\right)} \quad g=\frac{\chi^{2}}{2 J w} \quad s^{2}=\frac{V^{2}}{V_{a c}^{2}},
$$

Here $V$ is the velocity of soliton propagation, which is determined by the wave number:

$$
V=a \frac{d \zeta}{d t}=\frac{2 J a}{\hbar} \sin (Q)
$$

$V_{a c}$ is sound velocity in a chain, and $a$ is a lattice constant (the equilibrium distance between the nearest sites). The spatial distributions of charge and current in such a system are expressed via the wave functions (1) by the following relations

$$
\rho_{n}(t)=e \frac{\left|\psi_{n}(t)\right|^{2}}{\pi r_{0}^{2} a}, \vec{j}_{n}(t)=\vec{l} \frac{i e J}{\hbar \pi r_{0}^{2} a}\left(\psi_{n}^{*} \psi_{n+1}-\psi_{n+1}^{*} \psi_{n}\right)
$$

respectively.

\section{ELECTROMAGNETIC PROPERTIES OF ELECTROSOLITONS}

It has been shown by Brizhik et al. [11] that solitons have some characteristic frequency

$$
\omega_{S}=g \frac{V_{a c}}{\pi},
$$

with respect to which the external periodic EMF has a resonant effect on their dynamics. Namely, in the fields with low $\left(\omega<\omega_{s}\right)$ and high $\left(\omega>\omega_{s}\right)$ frequencies, solitons are stable, they oscillate with 
the frequency of the external filed $\omega$ as quasi-particles under the effect of external periodic force, while at the resonant frequency $\left(\omega=\omega_{s}\right)$ they absorb intensively energy from the field, and radiate it in the form of sound waves (vibrational modes in the chain) and electromagnetic radiation. This causes fast decay of electrosolitons, affecting, therefore, the efficiency of the redox processes in particular, and the whole charge transport in the metabolism of the system, in general.

In molecular chains, solitons expire periodic potential relief, caused by the discreetness of chains, according to Brizhik et al [12]. As a result, soliton velocity is an oscillating function of time:

$$
V(t)=V_{0}\left[1+\sum_{m=1}^{\infty} \frac{4 q^{m}}{1+q^{2 m}} \cos \left(m \omega_{0} t\right)\right],
$$

with the frequency of the main harmonic

$$
\omega_{0}=\frac{2 \pi V_{0}}{a}
$$

where $V_{0}$ is average velocity of the soliton per its period of oscillations. Therefore, solitons attain properties of breathers - nonlinear localized modes with internal oscillating (breathing) structure. Such an oscillating character of charge dynamics, with the nonzero acceleration $a d^{2} \zeta / d t^{2}$, according to Maxwell equations, is accompanied by emission of the electromagnetic field with the vector and scalar potentials determined by the charge and current distributions defined in eqn (6):

$$
\varphi(\vec{r}, t)=\frac{1}{4 \pi \varepsilon_{0}} \int \frac{\left[\rho\left(\vec{r}^{\prime}\right)\right]}{R\left(\vec{r}, \vec{r}^{\prime}\right)} d v^{\prime} \vec{A}(\vec{r}, t)=\frac{\mu_{0}}{4 \pi} \int \frac{\left[\vec{j}\left(\vec{r}^{\prime}\right)\right]}{R\left(\vec{r}, \vec{r}^{\prime}\right)} d v^{\prime},
$$

respectively. Here $\vec{r}$ is radius-vector, $R\left(\vec{r}, \vec{r}^{\prime}\right)$ is the distance between the corresponding points, the square brackets indicate that the inside functions account for the retardation effects, i.e. the functions are calculated at time moment

$$
t^{\prime}=t-\frac{R\left(\vec{r}, \vec{r}^{\prime}\right)}{c}
$$

Solution of Maxwell equations, according to Brizhik and Eremko [13], shows that in addition to the component of the field that is inversely proportional to the square of the distance (the field itself), there is also the component which decays as inversely proportional to the distance. This is the radiation component of the field slowly decaying in space with the intensities of the electric and magnetic field:

$$
\begin{gathered}
\vec{E}_{r a d}(r)=\frac{e V_{0}^{2}}{2 a \varepsilon_{0} c^{2} r^{3}} \sum_{n=1}^{\infty} \frac{4 n q^{n}}{1+q^{2 n}} \sin \left(n \omega_{0} t\right) \vec{r} \times(\vec{r} \times \vec{l}), \\
\vec{B}_{r a d}(r)=\frac{e V_{0}^{2}}{2 a \varepsilon_{0} c^{2} r^{2}} \sum_{n=1}^{\infty} \frac{4 n q^{n}}{1+q^{2 n}} \sin \left(n \omega_{0} t\right) \vec{l} \times \vec{r},
\end{gathered}
$$

respectively. Therefore, such a radiation is the superposition of harmonics with multiple frequensies, with the main harmonic $\omega_{0}$ determined in eqn (9).

The estimate of the main frequency of the radiation field, for instance, for polypeptide chains with the parameters given in Scott [14] gives $v=w_{0} / 2 \pi \sim 10^{12} \mathrm{~s}^{-1}$. Therefore, electrosolitons radiate high 
frequency electromagnetic waves in the millimeter interval of the wavelength, which agrees with the experimental data on the measurement of the endogeneous high-frequency EMFs (see Pohl [15], Pokorny et al. [16], Montagnier et al. [17], Cifra et al. [18], and references therein).

Such radiation has various important consequences. One of it is connected with the fact that the high-frequency alternating EMF of one of electrosolitons affects electrosolitons in other chains, and, thus, via such a field the electroslitons interact with each other, and this interaction is long-range. Such a mutual influence of electrosolitons via the self-induced radiation results in the synchronization of electrosoliton dynamics with time [13]. This synchronization of electrosoliton dynamics results in the tuning of their radiation frequencies, which are determined by the relations (9). Due to this, the radiation fields become coherent, therefore, the total intensity of such endogenous fields is proportional to the square of the total number of electrons in the system, $E \sim N_{e}^{2}$, and, therefore, is macroscopic value.

Another consequence of this result is the mechanism for the long-range exchange of the information between various systems, mediated by EMF, and appearance of the hierarchy of interacting systems: the electromagnetic potential can cause the emergence of the coherent structures that in view of their coherence, openness, and nonlinearity, are able to self-organize and form various hierarchical levels of ecosystems (Brizhik et al. [19]). In the meantime, the electromagnetic potential is also the physical agent acting as a messenger in these coherent structures, reducing the number of independent microstates of a coherent system, that can be compared with Schrödinger's concept of negentropy (see also Tiezzi [20]). Electromagnetic potential is essential for the self-organization and the time evolution of any complex system, including ecosystems, providing the mechanism for their non-locality, complexity, and self-consistency (self-maintaining), according to the above obtained results. Since an electromagnetic potential adds up to the phase of the system (Böhm - Aharonov effect - see Aharonov and Böhm [21,22]), the electromagnetic potential is able to drive the evolution of the phase that, in turn, drives the further evolution of the coherent system. As a consequence, the evolution of each coherent component of complex open system is affected by the electromagnetic potential produced by all other components. Such self-consistent non-local dynamics of matter and EMF provides development, or, as I. Prigogine writes, [23], metabolism of dissipative structures, increasing not only the network of individuals and processes, but also information.

\section{RATCHET DYNAMICS OF SOLITONS}

In this section we show that periodic in time EMF without a constant component (unbiased field) under certain conditions can cause a drift of electrosolitons. As it has been discussed in Section 2, one of the general features of charge transport systems, such as macromolecules, DNA, and water chains is that they can be modeled as two subsystems, charges (electrons or protons) and lattice, which play the roles of the potentials. Since unit cells of molecular lattices contain few atoms, such potentials in the general case are asymmetric and multi-well. For the purposes of this section we consider the simplest case of a diatomic chain with a double-well potential. In macromolecules and DNA this is the so-called Peierls-Nabarro potential [12], in water chains this is a relief, formed by double-well potentials for a proton in hydrogen-bonded water molecules [2, 8]. In all these cases such potential reliefs can play the role of the ratchet potential for the appearance of the ratchet phenomenon. Worth to recall that the term 'ratchet' means appearance of a directed current (drift) under the action of stochastic or deterministic unbiased (zero mean-value) ac forces (Reinmann, [24]. The ratchet phenomenon attracts an ever increasing attention due to its importance both for understanding of the functioning of biological motors and for promising technical applications in nanotechnologies [24]. This phenomenon can also take place in charge transport systems, described above in this paper, under the action of an external field, a local transmembrane potential in a living 
cell, a coherent electromagnetic field of the whole organism (endogenous electromagnetic field), etc. Since ratchet phenomenon can essentially affect dynamics of charged solitons (electrosolitons) and their stability properties, it can affect in its turn the metabolism of living organisms in particular and ecosystems in general.

In the general case soliton dynamics in a molecular lattice (macromolecule, hydrogen-bonded water chain, etc.) is described by the system of nonlinear coupled equations for the electron wave function and molecule displacements which can be reduced to eqn (1), which is the Schrödinger equation for the electron wave function in the self-consistent deformation potential, proportional to the electron probability. In the leading order approximation, this equation has the soliton solution (2)-(3). Let us denote masses of atoms $M_{1}$ and $M_{2}$; on-site electron levels $E_{1}$ and $E_{2}$, the elasticity constants describing the strengths of the interactions between, respectively, the nearestneighbour atoms belonging to one unit cell and to the neighbouring cells, $W_{s}$ and $W_{l}$; the corresponding hopping interactions $J_{s}$ and $J_{l}$, and, finally, the coefficients of the electron-phonon interaction between nearest neighbours $\chi_{s}$ and $\chi_{l}$. It is convenient to introduce the anisotropy parameters:

$$
m=\frac{M_{1}-M_{2}}{M}, m=\frac{M_{1}-M_{2}}{M}, d=\frac{J_{s}-J_{l}}{J}, x=\frac{\chi_{s}-\chi_{l}}{\chi}
$$

where $M=M_{1}+M_{2}, W=w_{s}+w_{l}, J=J_{s}+J_{l}, \chi=\chi_{s}+\chi_{l}$.

Width of the soliton function (3) in a diatomic chain is given by the relation:

$$
\mu=\frac{4 G^{2}\left(1+x^{2}-2 x w\right) \sqrt{1+\frac{D^{2}}{4}}}{a C\left(1-d^{2}\right)\left(1-w^{2}\right)},
$$

where the notations are used:

$$
G=\frac{X l}{2 J}, C=\frac{\hbar^{2} W}{M J^{2}}, D=\frac{E_{2}-E_{1}}{J}
$$

In Fig. 1 we show the distribution of the electron probability (1) as function of the lattice site for the parameter values $G=0.4, C=0.22, d=D=0.1, x=0.05, m=0.3$.

A soliton described by the wave function (3), can be viewed as a single particle with the c.m. coordinate of the soliton, $\varsigma(t)$. In a molecular chain the dynamic equation for the c.m. coordinate of the soliton, $R(t)$, under the external force, $F(t)=e E(t)$, taking into account the lattice discreteness and the energy dissipation, takes the form:

$$
M_{s} \ddot{\varsigma}=\gamma \dot{\varsigma}+f(\varsigma)+e E(t)
$$

where $M_{S}$ is the effective mass of a soliton, 'dressed' with phonons, $\gamma$ is the friction coefficient, proportional to the constant of energy dissipation in the system, and the force $f(\varsigma)=-d U(\varsigma) / d \varsigma$ comes from the Peierls-Nabarro potential $U=U_{P N[12]}$ or from a relief of double-well potentials in water chain $U=U_{w}[2,8]$. Both potentials are spatially periodic with the period of the lattice constant, $U(\varsigma)=U(\varsigma+a)$. It is well known that equations of the type (17) can lead to the ratchet phenomenon [24], i.e. to a unidirectional (in average) motion (ratchet dynamics) of a particle whose trajectory is a limit cycle phase locked to the external periodic drive $E(t)$.

In a diatomic molecular chain, the potential relief can be written as

$$
U(R)=U_{1} \cos (2 \pi \varsigma / a)+U_{2} \cos (4 \pi \varsigma / a+\Theta),
$$




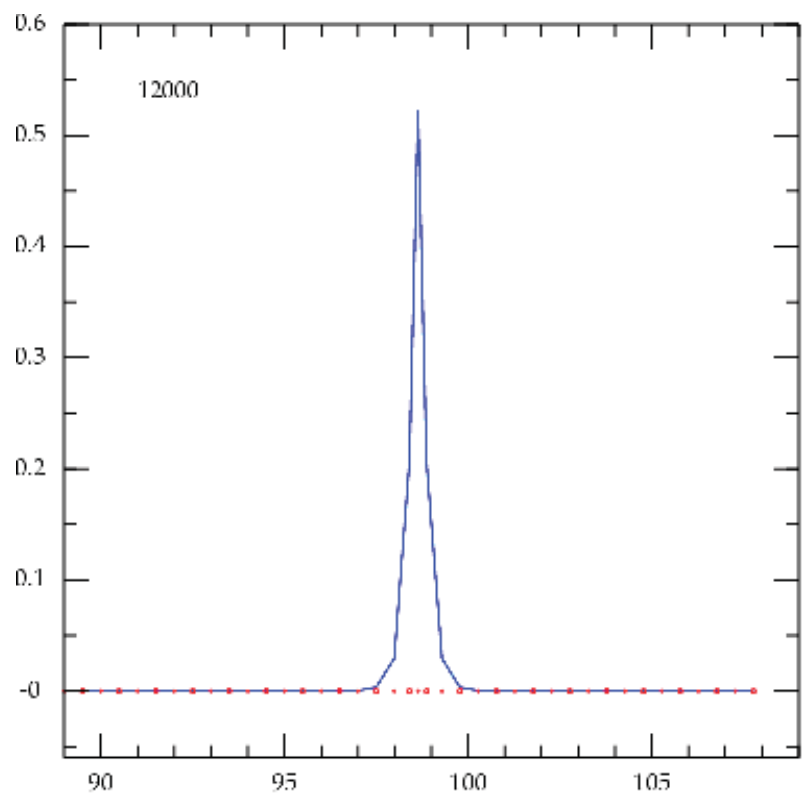

Figure 1: Stationary distribution of the electron probability amplitude (3) as function of the lattice site $n$. The parameter values are given in the text.

where $\Theta$ is a phase that determines the asymmetry of the potential arising from the difference of atoms in a unit cell. The height of the barrier depends on the square of the electron-phonon coupling, similarly to the case of a uni-atomic chain [12]. Namely, the presence of such a potential causes the possibility of the charge drift in the unbiased field, provided its intensity and period exceed some critical values, as has been shown by computer modeling of the initial system of discrete equations (Brizhik et al., [25]) and as it can be seen by solving eqn (17) with the potential given by eqn (18) for a periodic unbiased field $E(t)=E_{0} \sin (2 \pi t / T)$ numerically. An example of such a drift is shown in Fig. 2 for the intensity of the field $E_{0}=0.08$ and period of the field $T=2000$. Here the intensity of the field is measured in units $e a / J$ and time in units of $\hbar / J$.

Of course, the average velocity of the soliton strongly depends on the chain parameters, energy dissipation, and parameters of the driving force, $E(t)$. Moreover, the ratchet phenomenon belongs to the class of critical phenomena: it appears, providing the intensity of the field and its period exceed certain critical values for the given values of the parameters of the system. The existence of the threshold with respect to the intensity of the field and its period can be explained using the results of the study of the dynamics of solitons in external field, considered in Section 2 (see also [11]), according to which solitons have some characteristic frequency $\omega_{s}$ defined in eqn (7). Under the action of the periodic field, solitons oscillate with the frequency of the applied field, $\omega$, but their effective mass depends on the frequency of the field. Namely, in low-frequency regime $\left(\omega<\omega_{s}\right)$ deformation of the chain follows oscillations of a charge, and soliton effective mass includes the mass of the chain deformation. In a high-frequency regime $\left(\omega>\omega_{s}\right)$, the deformations remains at rest and soliton effective mass equals to the effective mass of a free charge. Since for the appearance of the ratchet dynamics the presence of the ratchet potential is a necessary condition, one can expect, that such ratchet dynamics can be possible in low-frequency regime, which agrees with the numerical simulations. Moreover, for driving amplitudes below the threshold (depinning threshold), the soliton remains pinned to the lattice and its c.m. oscillates around the lattice site. The drift is possible 


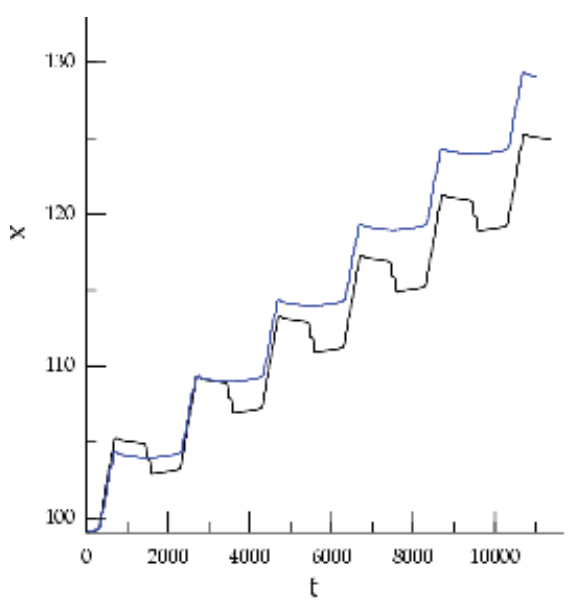

Figure 2: Position of the c.m. of the soliton, $x$, as function of time, $\mathrm{t}$, in an external harmonic field $E_{0}=.008, T=2000$ in a chain with the parameter values $G=.4, C=.22, d=D=.1$ at different anisotropy values: $x=.05, m=.3, w=.15$ (bottom) and $x=w=m=0$ (top).

when the intensity of the field exceeds the depinning threshold and the amplitude of the soliton oscillations exceeds the lattice constant (the period of the potential barrier).

\section{CONCLUSIONS}

Electrosolitons, which are the charge, energy, and information carriers in the metabolic processes in biological systems, according to Davydov's hypothesis [2], are formed in alpha-helical proteins, DNA, and hydrogen-bonded water molecule chains, present in living cells, intercellular tissues, and in all ecological systems. The results reported here show that electromagnetic field can affect significantly charge transport process in living organisms and ecosystems. In the external fields at frequencies which do not coincide with the characteristic frequency of solitons (7), solitons remain stable, but their dynamic mass depends on the frequency of the field. At the resonant frequency, solitons intensively emit radiation and sound waves and, losing their energy, can be destroyed at all. In both cases of resonant and non-resonant regimes, such changes in the dynamics of solitons induced by the external field, of course, affect charge transport processes in particular and the metabolism of the system in general.

During propagation along the corresponding molecular chains, electrosolitons emit their own electromagnetic radiation with the frequency of the main harmonic (9) which depends on their velocity, i.e. on the (bio)chemical and physical processes in the corresponding system. Via this electromagnetic radiation, subunits of the complex system (cells, organs, individuals, ecosystems) exchange their information which leads to the appearance of the long-range order. It is essential that not only electric and magnetic components of the field accompany the evolution of the system, but that such evolution is affected by self-induced scalar and vector-potentials. According to the above, these potentials are self-consistent with the metabolism of the system and are responsible for the self-organization of complex systems and emergence of coherence. Vector-potentials are produced by every fluctuating charged object but can be detected only by coherent systems at their resonant frequencies and attain macroscopic intensity due to the 'antenna effect' because of the synchronizing effect of the radiation on dynamics of electrosolitons and tuning of the frequencies of the radiation components of individuals. The vector-potential has no energy content, it propagates at the phase 
velocity that is not bound by the speed of light. As a result, such self-consistent dynamical selforganization and coherence can evolve into the hierarchy of dissipative systems able to support long-range communication at high speed. As I. Prigogine has indicated, if an ecosystem works perfectly, it increases its own network and information [23]. Namely, the electromagnetic interaction provides the most powerful mechanism for the fastest long-range exchange of the information and interconnectedness (non-locality) of ecosystems.

In the external electromagnetic field at certain conditions, as it has been discussed in Section 4, electrosolitons can attain ratchet properties and drift in non-biased fields. Soliton drift takes place due to the absorption of energy from the external field. During the oscillations in the periodic in time field solitons emit sound waves in both directions and the amplitude of the deformational field, corresponding to this emission of sound waves, is proportional to the frequency and intensity of the field. Similar to the deterministic fields considered here, white noise [26, 27] also can cause unidirected current of solitons in molecular systems and uni-directed drift of charges in hydrogen-bonded water structures. In the latter case, under certain conditions the corresponding currents can be macroscopic, like, for instance, in macroscopically large coherence domains of water, which can exist in clouds. We dare to suggest a hypothesis, that namely in this way the lightning is formed in clouds. On the other hand, this study suggests a possible mechanism of the effects of the solar activity on biosystems via its influence on the nonlinear charge transport processes, as well it indicates a possible danger of technologically produced electromagnetic smog even of low intensities in view of the resonant nature of such bioeffects.

\section{ACKNOWLEDGMENT}

I express my sincere gratitude to E. Del Giudice and J. Pokorny for the highly stimulating discussions on the subjects of this paper. I also acknowledge my colleagues L. Cruzeiro, A. Eremko, B. Piette, and W. Zakrzewski for the productive joint research, some of the results of which were reported in this paper. I dare to dedicate this work to the memory of Enzo Tiezzi, whom I was privileged to meet and whose interdisciplinary background and enthusiasm have stimulated my interest to nonlinear dynamics of ecosystems.

\section{REFERENCES}

[1] Lehninger, A.L., Biochemistry, Worth Publishers Inc.: New York, 1972.

[2] Davydov, A.S., Solitons in Molecular Systems, 2nd edn, Reidel: Dordrecht, 1985.

[3] Dauxois, T. \& Peyrard, M., Physics of Solitons, Cambridge University Press: Cambridge, 2006.

[4] Antonchenko, V.Ya., Davydov, A.S. \& Zolotaryuk, A.V., Solitons and proton motion in icelike structures. Physica Status Solidi B, 115, pp. 945-952, 1983. doi:http://dx.doi.org/10.1002/ pssb.2221150234

[5] Pollack, G.H., Cells, Gels and Engines of Life, Ebner \& Sons: USA, 2001.

[6] Voeikov, V.L., Fundamental role of water in bioenergetics. Biophotonics and Coherent Systems in Biology, eds L.V. Beloussov, V.L. Voeikov \& V.S. Martynyuk, Springer: New York, pp. 89-98, 2007.

[7] Huang, C., The inhomogenous structure of water at ambient conditions. PNAS, 106, pp. 15214-15218, 2009. doi:http://dx.doi.org/10.1073/pnas.0904743106

[8] Del Giudice, E., Spinetti, P.R. \& Tedeschi, A., Water dynamics at the roots of the metamorphosis in living organisms. Water, 2, 2010; doi:http://dx.doi.org/10.3390/w20x000x.

[9] Preparata, G., QED Coherence in Matter, World Scientific: New Jersey, 1995. doi:http://dx.doi. org/10.1142/9789812830999 
[10] Del Giudice, E., De Ninno, A., Fleischmann, M., Mengoli, G., Milani, M., Talpo, G. \& Vitiello, G. Coherent quantum electrodynamics in living matter. Electromagnetic Biology and Medicine, 25, pp. 522-530, 2006.

[11] Brizhik, L., Cruzeiro-Hansson, L. \& Eremko, A., Influence of electromagnetic radiation on molecular solitons. Journal of Biological Physics, 24, pp. 19-39, 1998. doi:http://dx.doi. org/10.1023/A:1005096714234

[12] Brizhik, L., Cruzeiro-Hansson, L., Eremko, A. \& Olkhovska Yu., Soliton dynamics and Peierls-Nabarro barrier in a discrete molecular chain. Physical Review B, 61, pp. 1129-1141, 2000. doi:http://dx.doi.org/10.1103/PhysRevB.61.1129

[13] Brizhik, L.S. \& Eremko, A.A., Nonlinear model of the origin of endogenous alternating electromagnetic fields and selfregulation of metabolic processes in biosystems. Electromagnetic Biology and Medicine, 22, pp. 31-39, 2003. doi:http://dx.doi.org/10.1081/JBC-120020351

[14] Scott, AC., Davydov's soliton. Physics Reports, 217, pp. 1-67, 1992. doi:http://dx.doi. org/10.1016/0370-1573(92)90093-F

[15] Pohl, H.A., Oscillating fields about growing cells. International Journal of Quantum Chemistry. Quantum Biology Symposium, 7, pp. 411-431, 1980.

[16] Pokorný, J., Hašek, J. \& Jelínek, F., Endogenous electric field and organization of living matter. Electromagnetic Biology and Medicine, 24, pp. 185-197, 2005. doi:http://dx.doi. org/10.1080/15368370500379566

[17] Montagnier, L., et al., Electromagnetic signals are produced by aqueous nanostructures derived from bacterial DNA sequences. Interdisciplinary Sciences: Computational Life Sciences, 1, pp. 81-90. 2009.

[18] Cifra, M., Fields, J.Z. \& Farhadi, A., Electromagnetic cellular interactions. Progress in Biophysics and Molecular Biology, pp. 1-24, 2010. doi:http://dx.doi.org/10.1016/ j-pbiomolbio.2010.07.003

[19] Brizhik, L., Del Giudice, E., Jorgensen, S.E., Marchettini, N. \& Tiezzi, E., The role of electromagnetic potentials in the evolutionary dynamics of ecosystems. Ecological Modelling, 220, pp. 1865-1869, 2009. doi:http://dx.doi.org/10.1016/j.ecolmodel.2009.04.017

[20] Tiezzi, E., Steps Toward an Evolutionary Physics, WIT Press: Billerica, MA, 2005.

[21] Aharonov, Y. \& Böhm, D., Significance of electromagnetic potentials in quantum theory. Physics Review, 115, pp. 485-491, 1959. doi:http://dx.doi.org/10.1103/PhysRev.115.485

[22] Aharonov, Y. \& Böhm, D., Further considerations on electromagnetic potentials in the quantum theory. Physics Review, 123, pp. 1511-1524, 1961. doi:http://dx.doi.org/10.1103/ PhysRev.123.1511

[23] Prigogine, I. \& Nicolis, G., Self-Organisation in Non-Equilibrium System, From Dissipative Structures to Order Through Fluctuations, Wiley: New York, 1977.

[24] Reinmann, P., Physics Reports, 361, pp. 57-121, 2002. doi:http://dx.doi.org/10.1016/ $\underline{\text { S0370-1573(01)00081-3 }}$

[25] Brizhik, L., Eremko, A., Piette, B. \& Zakrzewski, W., Ratchet dynamics of large polarons in asymmetric diatomic molecular chains. Journal of Physics: Condensed Matter, 22, 155105, 2010; http://stacks.iop.org/0953-8984/22/155105. doi:http://dx.doi.org/10.1088/ $\underline{0953-8984 / 22 / 15 / 155105}$

[26] Luczka, J., Bartussek, R. \& Hänggi, P., Europhysics Letter., 31, pp. 431-435, 1995. doi:http:// dx.doi.org/10.1209/0295-5075/31/8/002

[27] Luczka, J., Chernik, T. \& Hänggi, P., Physics Review E, 56, pp. 3968-3974, 1997. doi:http:// dx.doi.org/10.1103/PhysRevE.56.3968 